\title{
Responsabilidad social de las universidades latino-americanas
}

\section{Social responsibility of Latin American universities}

DOI: $10.46932 / \mathrm{sfjdv} 2 \mathrm{n} 5-133$

Received in: Oct 1st, 2021

Accepted in: Dec 30th, 2021

\author{
Carlos Rios-Campos \\ Investigador RENACYT.
}

Doctor en Gestión Universitaria. Maestro en Administración.

Ingeniero de Sistemas.

Docente de la Universidad Nacional Toribio Rodríguez de Mendoza de Amazonas.

Miembro del Colegio de Ingenieros del Perú. Section Investigation Activities Chair North (IAC) - IEEE Perú.

Calle La Plata 155 - Urb: San Eduardo - Chiclayo, Perú.

E-mail: carlos.rios@untrm.edu.pe

\section{Norma Alexandra Hinojosa Garcés}

Docente del Nivel Superior

Magíster en Gerencia y Liderazgo Educacional

Av. De las Américas frente al Cuartel Modelo. Dirección domiciliaria: La Joya Etapa Esmeralda.

E-mail: nhinojosag@ulvr.edu.ec

\section{Alba Jazmín Morán Mazzini \\ Docente del Nivel Superior \\ Magíster en Pedagogía}

Av. De las Américas frente al Cuartel Modelo.

Floresta 2 Mz 194. Código Orcid: 0000-0002-8187-373X

E-mail: amoranm@ulvr.edu.ec.

\section{Janet Avalos Hubeck}

Mg. Gestión de Servicios de Salud.

Lic en Enfermeria. Docente de la Universidad Señor de Sipán.

Olimpo 129 - Urbanización El Paraíso - Chiclayo, Perú.

E-mail: javalosh@crece.uss.edu.pe

\section{Enrique Guillermo Llontop Ynga}

Médico Cirujano.

Magister en Gestión de Servicios de Salud, Universidad Señor de Sipán, Universidad Cesar Vallejo.

Olimpo 129 - Urbanización El Paraíso - Chiclayo, Perú.

E-mail: ellontop@crece.uss.edu.pe / ellontopy@ucvvirtual.edu.pe

Daniel Jesús Castro Vargas

Magister en Administración de la Educación

Licenciado Administración de Empresas

Contador Público Colegiado

Director del Instituto de Investigación de Desarrollo Social de la Universidad Nacional Autónoma de Chota - UNACH. 
Diego Palomino N 1618 - Jaén - Cajamarca, Perú.

E-mail: djcastrov@unach.edu.pe

Irma Rumela Aguirre Zaquinaula

Doctora en Administración de la Educación

Docente Ordinaria Principal de la Universidad Nacional de Jaén.

Jirón Cuzco 250 - Jaén, Perú.

E-mail: irma.aguirre@unj.edu.pe.

Giuliana Fiorella Samame Aguirre

Médico cirujano, Especialista en Anestesiología

Maestra en Gestión de los Servicios de Salud, Maestra en Medicina con Mención en Cirugía.

Hospital Leoncio Prado.

Av 10 de julio 209 - Huamachuco, Perú.

E-mail: giulianasamame31@gmail.com

\title{
RESUMEN
}

En la gestión universitaria actual, la responsabilidad social universitaria (RSU) es un aspecto muy importante. En esta investigación, se planteó el objetivo general, analizar la responsabilidad social de las universidades latinoamericanas. Metodología, la investigación presentó un diseño cualitativointerpretativo, de tipo documental, se han seleccionado 50 documentos, realizados en el periodo 2016 2021, incluyendo: artículos científicos, artículos de revisión e información de sitios web de organizaciones reconocidas. Las palabras claves utilizadas en las búsquedas fueron: Responsabilidad social universitaria, Latinoamérica, universidad. Resultados, se observa un esfuerzo permanente en el desarrollo de la responsabilidad social de las universidades latinoamericanas, mediante la formación de redes, asociaciones, eventos académicos y priorización de la RSU en sus objetivos institucionales. Conclusiones, La responsabilidad social universitaria (RSU) es importante por su impacto en la sociedad. Debe formar parte de las estrategias universitarias latinoamericanas y ser protagonistas de la mejora social. La gestión universitaria actual no debe descuidar la RSU. Han surgido diversas redes y asociaciones latinoamericanas que sirven de impulso de la RSU, por lo que se recomienda una mayor integración de las universidades públicas y privadas, para crear sinergias. En cada país latinoamericano analizado, destacan algunas universidades que lideran la RSU y que deberían ser imitadas, por los resultados positivos que están logrando. Se recomienda concientizar y capacitar al personal docente y administrativo en la RSU, para lograr un mayor compromiso social.

Palabras clave: Responsabilidad social universitaria, Latinoamérica, universidad.

\begin{abstract}
In current university management, university social responsibility (USR) is a very important aspect. In this research, the general objective was set: to analyze the social responsibility of Latin American universities. Methodology, the research presented a qualitative-interpretive design, documentary type, 50 documents have been selected, made in the period 2016 - 2021, including: scientific articles, review articles and information from websites of recognized organizations. The keywords used in the searches were: University social responsibility, Latin America, university. Results, a permanent effort is observed in the development of social responsibility of Latin American universities, through the formation of networks, associations, academic events and prioritization of USR in its institutional objectives. Conclusions, University social responsibility (RSU) is important for its impact on society. It must be part of Latin American university strategies and be protagonists of social improvement. Current university management should not neglect RSU. Various Latin American networks and associations have emerged
\end{abstract}


that serve as the impetus for USR, for which a greater integration of public and private universities is recommended, to create synergies. In each Latin American country analyzed, there are some universities that lead the USR and that should be imitated, due to the positive results they are achieving. It is recommended to raise awareness and train teaching and administrative staff in RSU, to achieve greater social commitment.

Keywords: University social responsibility, Latin America, university

\section{INTRODUCCIÓN}

En esta investigación se planteó el objetivo general, analizar la responsabilidad social de las universidades latinoamericanas.

\subsection{RESPONSABILIDAD SOCIAL UNIVERSITARIA (RSU)}

Entender la universidad como uno de los agentes de cambio de gran responsabilidad en la sociedad es reconocerla más allá de su labor educativa, en cuanto al trabajo de generar conocimientos científicos y académicos, es ver la universidad como una institución de interacción en la que se desarrollan procesos desde las diferentes áreas del saber...(Barreto et al., 2021).

La Universidad está llamada a convertirse en la institución docente y científica principal en los territorios, ya que es capaz de construir conexiones para la circulación del conocimiento entre las instituciones y el resto de los actores, acercando los procesos académicos a las necesidades y demandas de cada localidad y creando las premisas para elevar la pertinencia y el impacto universitario en la sociedad

(Medina et al., 2017).

La RSU debe formar parte de las estrategias diseñadas por las instituciones de educación superior, con la consiguiente asignación de recursos, pero no puede olvidarse que esa determinación debe proyectarse al exterior, a la comunidad que se ve afectada, de distinta forma, por las actuaciones desarrolladas por las universidades y los correspondientes impactos generados (Aldeanueva \& Arrabal, 2018).

Los resultados permitieron identificar tres perspectivas: medición, estratégico y conexión; también muestran que este campo de estudio es relativamente joven, en el cual el autor principal es Ricardo Gaete, mientras que François Vallaeys es el más citado. Adicionalmente, la región de mayor producción en el tema es Suramérica (Duque \& Cervantes-Cervantes, 2019).

La Responsabilidad Social Universitaria (RSU) cada día cobra mayor importancia en la gestión universitaria. 


\subsection{UNIVERSIDADES LATINOAMERICANAS}

La influencia del movimiento estudiantil de Córdoba en 1918 en otras universidades latinoamericanas, como en Lima - Perú, La Habana - Cuba, o en la ciudad de México llevó a reformas profundas en las universidades, anticlericales y antiimperialistas y por supuesto por una mayor participación de los estudiantes en los asuntos universitarios y a favor de una independencia de las instituciones de educación superior de las ingerencias de los gobiernos, a favor de una autonomía universitaria (Marsiske \& Vera de Flachs, 2018).

Los aportes más significativos y comunes de todos los movimientos reformistas en América Latina, en esencia pretendían que la universidad pública aportara a la sociedad a partir de asumir su función social o mejor dicho, la responsabilidad social que le corresponde, que significa, en opinión de los estudiosos del tema, poner el saber universitario al servicio de la sociedad y hacer de sus problemas un aspecto importante de su ocupación; función que, como se puede apreciar, es consecuencia de un proceso histórico orientado a lograr la apertura y democratización de la universidad y su más amplia proyección social (González \& Fernández- Larrea, 2021).

La investigación realizada permitió identificar algunas variables clave a considerar por las instituciones de educación superior latinoamericanas con el fin de lograr una efectiva influencia de la internacionalización en los procesos universitarios, tales como: globalización de la educación superior, cambios estructurales en la universidad e internacionalización como proceso estratégico institucional (Llano et al., 2021).

Uno de los retos que enfrentan las universidades latinoamericanas es diseñar e implementar estrategias institucionales acordes a los marcos nacionales e internacionales basados en el respeto por los derechos humanos de las mujeres y por la promoción de sociedades libres de violencia de género. Las universidades deberían liderar estos procesos. Sin embargo, parecen estar aún lejos de ese protagonismo (Serna et al., 2018).

Las universidades latinoamericanas tienen que superar muchos retos pendientes, sobre todo durante la pandemia y establecer un mayor vínculo con la sociedad para ayudarla a resolver los problemas.

\section{METODOLOGÍA}

La investigación presenta un diseño cualitativo-interpretativo, de tipo documental, el cual precisó el procedimiento de selección y el registro de los datos (Barrero y Rosero, 2018).

En la presente investigación se han seleccionado 50 documentos, realizados en el periodo 2016 2021; incluyendo: artículos científicos, artículos de revisión e información de sitios web de organizaciones reconocidas. Las palabras claves utilizadas en las búsquedas fueron: Responsabilidad 
social universitaria, Latinoamérica, universidad. Para la selección de los documentos se usaron como criterios: el año de la publicación, la pertenencia con la investigación y ser una fuente confiable. Después de la lectura de cada documento, se procedió a ingresar los datos a la matriz bibliográfica, que sirve para catalogar los documentos de acuerdo con categorías, que se presentan en la tabla 1.

Tabla 1. Matriz bibliográfica

\begin{tabular}{|l|l|l|l|}
\hline Nombre & Tipo & Objetivos & Conclusiones \\
\hline & & & \\
\hline & & & \\
\hline
\end{tabular}

Fuente: Adaptado de Barrero y Rosero (2018).

\section{RESULTADOS}

Se muestra y analiza a continuación la responsabilidad social de las universidades latinoamericanas.

\section{MÉXICO}

La Universidad Autónoma del Estado Morelos (UAEM) es la institución de educación superior pública del país que,... En virtud de lo anterior, consideramos que el caso estudiado revela una enorme contradicción en relación con la RSU o USR, ya que los hechos evidencian que las autoridades de la universidad, lejos de considerar y prever los impactos sociales y asumir una verdadera responsabilidad más allá de la retórica, han hecho exactamente lo contrario: perjudicar a la institución y a los diversos actores que convergen en ella al hipotecar su desarrollo, prestigio y sustentabilidad (Ibarra et al., 2020).

Se destaca la participación de las facultades de negocios, adscritas a la dirección general del área académica económico administrativa de la Universidad Veracruzana, favoreciendo al fortalecimiento de la responsabilidad social, en el que a partir de este referente, se perciben indicadores de evaluación, crecimiento y gestión de buenas prácticas en las entidades académicas para la toma de decisiones, y con ello propiciar, promover y coordinar un plan de mejora a partir del autoconocimiento para un impacto social (Nieto \& Gazca, 2021).

\section{GUATEMALA}

Por otra parte, desde el impacto organizacional de la responsabilidad social, se promovió el cuidado de la salud integral de la comunidad Landivariana a través de la implementación de protocolos de salud en el Campus Central y los distintos Campus y Sedes. En estos proyectos y acciones se ha contado con la participación de docentes, estudiantes, personal administrativo y proveedores son una muestra de la esencia de la Universidad Rafael Landívar (Universidad Rafael Landívar, 2020). 


\section{REPÚBLICA DOMINICANA}

Durante su exposición, el doctor Vallaeys explicó que la responsabilidad social es una nueva política de gestión que termina en los impactos sociales ambientales de las IES que generan en su entorno a través de sus procesos de formación, investigación y extensión para reforzar la participación de la educación superior en los desafíos que representan para cada país el cumplimiento de los ODS o Agenda de Desarrollo 2030 (RED COMUNICA, 2021).

La Pontificia Universidad Católica Madre y Maestra (PUCMM) formalizó su incorporación a la Red de Responsabilidad Social Universitaria de la Organización de Universidades Católicas de América Latina y el Caribe (ODUCAL), organismo que promueve y fortalece el desarrollo de experiencias de responsabilidad social universitaria como eje transversal de la formación católica universitaria y la ejecución de proyectos interinstitucionales de investigación en este campo (PUCMM, 2019).

\section{COSTA RICA}

A manera de ejemplo y sin pretender hacer una comparación exhaustiva, se logró ubicar que la Universidad Latina tiene la Unidad de Gestión del Trabajo Comunal Universitario creada a partir del 2014, pero no hay información sobre extensión o RSU (Universidad Latina de Costa Rica, s.f., s.p.). Por su lado, la Universidad Internacional de las Américas también tiene algo de información del TCU en el sitio web (Universidad Internacional de las Américas, s.f, s.p.), mientras que en la Universidad Fidélitas y la Universidad Hispanoamericana no se menciona nada relacionado con instancias, programas o proyectos de RSU o temas relacionados, ni siquiera del TCU (Lorena, 2019).

La Universidad de Costa Rica, al igual que otras universidades públicas o privadas, deben enfrentar el reto no solo de hacer una definición del término de RSU propia y ajustada a sus particularidades, sino de satisfacer una segunda necesidad, la cual consiste en incorporar este concepto dentro de sus políticas internas (plan estratégico y políticas internas, entre otros) (Herrera, 2019).

\section{PUERTO RICO}

Por ello, este estudio analiza las perspectivas de públicos internos sobre las iniciativas y procesos de responsabilidad social en una universidad privada en Puerto Rico...Se aprecia que los diversos públicos internos están conscientes de lo que significa, en la teoría y en la práctica, ser una universidad socialmente responsable aunque también reconocen oportunidades de mejora y desafíos futuros con respecto a la RSU (Gómez et al., 2018).

La responsabilidad social en la Universidad de Puerto Rico mediante la presentación de dos programas particulares...El primero es el caso del Programa de Investigación y Desarrollo Cooperativa (Incubadora) del Instituto de Cooperativismo de la Universidad de Puerto Rico y, el segundo, el Proyecto 
de alfabetización de adultos del Centro de Acción Urbana, Comunitaria y Empresarial de Río Piedras (CAUCE) (Baca y Reyes, 2019).

\section{HONDURAS}

El estado actual en Honduras con respecto al tema de RSU, en los 21 centros educativos superiores existentes, es que en 10 de ellos no se evidencian estructuras organizativas relacionadas con la extensión universitaria, la vinculación universidad-sociedad, del resto (11), 7 de ellas tienen programas y estructuras organizacionales de extensión universitaria, 3 de ellas tienen acciones denominadas y basadas en el enfoque de Vinculación Universidad-Sociedad, una de ellas destaca por disponer desde el 2007 un programa de Responsabilidad Social Universitaria (RSU), se trata de la Universidad Tecnológica de Honduras (UTH) (Banegas, 2018).

Como universidad comprometida y responsable con la sociedad, Zamorano se proyecta este 2020 con las comunidades vecinas al campus. Se ha entregado ayuda social y ha generado conocimientos que promuevan el progreso y crean capital social en favor del desarrollo y la calidad de vida de los pobladores de las zonas de escasos recursos (Universidad Zamorano, 2020).

\section{EL SALVADOR}

La docente de la Universidad de El Salvador (UES), Lourdes Elizabeth Prudencio, propuso en el marco del IX Congreso Universitario Centroamericano con sede en Honduras, redefinir la función social de las universidades con su entorno e institucionalizar la Responsabilidad Social Universitaria (RSU) como un nuevo paradigma de gestión obligada a responder el llamado de los demás como pago a una deuda social permanente, que además las obliga a reflexionar y poner en tela de juicio su papel y el de la ciencia (Vargas, 2021).

La Universidad Pedagógica de El Salvador "Dr. Luis Alonso Aparicio", forma parte de las 41 Instituciones de Educación Superior que integran la "Mesa de Responsabilidad Social Universitaria" lanzada recientemente, en el cual también es participe el Ministerio deEeducación Ciencia y Tecnología, así como otros actores de la Sociedad (Universidad Pedagógica de El Salvador, 2021).

\section{NICARAGUA}

Los universitarios quieren asumir su papel como sujetos sociales y políticos activos, para involucrarse en la toma de decisiones sobre aspectos de su interés, y no únicamente que los representantes estudiantiles sean quienes tomen las decisiones. Lo anterior es uno de los hallazgos que se desprenden del estudio denominado: “Cultura política y juventudes: Acción política de jóvenes en 
universidades públicas y privadas del CNU”, realizado por un equipo interdisciplinario del Centro Interuniversitario de Estudios Latinoamericanos y Caribeños, CIELAC-UPOLI (UPOLI, 2016).

En la Universidad Católica Redemptoris Mater (UNICA), la Responsabilidad Social Universitaria en nuestra alma máter exige, desde una visión holística, articular las diversas partes de la institución en un proyecto de promoción social de principios éticos y de desarrollo social equitativo y sostenible, para la producción y transmisión de saberes responsables y la formación de profesionales igualmente responsables y como nuestra misión lo indica que sean capaces de dar soluciones pertinentes a los retos que plantea Nicaragua en el contexto actual (UNICA, 2020).

\section{PANAMÁ}

Se concluye que fue posible articular ambos ejes de política universitaria y que el proyecto "Fortaleciendo los Planes y Programas de Estudio" propuesto desde la política de RSU, se potenció en el Proyecto de Transformación Académico-Curricular (TAC). A través de esta articulación se orientó una propuesta curricular, tendiente a alcanzar el desarrollo de habilidades sociales, expresadas en la relación con la sociedad, la educación para la conciencia social y el entendimiento de lo global y la exploración del mundo real...(Méndez, 2020).

Se analiza la realidad en materia de RSU en la que se encuentra la Universidad de Panamá, y aprovechando la corriente informativa que se genera en el proceso de Evaluación y Acreditación Institucional que se lleva a cabo en la citada Universidad, así como el apoyo de los Planes de Desarrollo Institucional, se han seleccionado una serie de indicadores de la Matriz de Acreditación, de los factores de docencia universitaria, de investigación e Innovación y de Extensión y se les ha dado un perfil específico que permita visualizar el valor RSU (Inmaculada, Montserrat y Abril, 2019).

\section{CUBA}

Se puede afirmar que la formación de la competencia investigativa en el PUN (profesor universitario novel) como parte de la RSU es un aspecto fundamental para el logro de una mayor conciencia, compromiso y sensibilidad de los profesores en el enfrentamiento de los problemas sociales que se presentan actualmente. Es esencial que la evaluación de la competencia investigativa, como parte de la RSU, pueda convertirse en una alternativa para unificar criterios sobre el desarrollo del PUN (González, 2017).

Fundamento de la responsabilidad social universitaria debe ser la formación integral -científica y humanista- de los individuos que permita la investigación, el diagnóstico y diseño de estrategias de gestión sociocultural, no solo como recurso para la preservación de las identidades nacionales o grupales, 
sino como alternativa para la solución de los conflictos y las necesidades espirituales que se generan en toda formación social; con ello se contribuye con el fomento de los valores (Valdés \& Villegas, 2017).

\section{COLOMBIA}

Para ello se desarrolló un estudio de caso en la Universidad del Valle...Con lo anterior se evidenció la contribución de la extensión universitaria al desarrollo social y se diseñaron acciones que aportan a su mejoramiento en pro de fortalecer la interacción universidad-sociedad (Mosquera-Abadía, \& Carvajal-Ordoñez, 2021).

El modelo propuesto parte de los cuatro pilares de la educación superior: docencia, proyección social, investigación y gestión, sumando medio ambiente y posconflicto... Se concluye que una de las causas de la no implementación de la RSU, es que no se cuenta con una metodología o un modelo de cómo hacerlo, así como al escaso interés prestado por las universidades; esto debido a que no se ha tomado consciencia de su importancia y lo favorable que resulta para contribuir a la reconstrucción del tejido social (Forero-Jiménez, 2019).

\section{VENEZUELA}

Analizar la responsabilidad social universitaria desde una visión prospectiva desde las funciones de docencia, investigación y extensión en la Universidad Centroccidental Lisandro Alvarado, Venezuela...Se concluyó que la racionalidad estratégica de las funciones universitarias, debe ser un eje transversal en la formación académica, con miras a desarrollar una prospectiva sistémica, sin barreras disciplinarias para asumir una actitud desestructurada ante la dinámica compleja de los avances científicos, tecnológicos, organizacionales y laborales, en procura de una mayor competitividad en el contexto que se vislumbra dentro del siglo XXI (Anchieta-Barrios y Pernía, 2018).

Se revisan las relaciones entre la RSU, Transferencia Tecnológica y Desarrollo Endógeno, Consejos Comunales, Comunidades y Estrategia de Vinculación Universitaria, dentro de los cinco objetivos históricos del Plan Patria de Venezuela (2013-2019)... Los resultados revelan que las universidades deben formular planes, programas y acciones estratégicas; así como los contactos previos con los grupos de interés que representan las ideas del colectivo, que involucran las alternativas reales de solución para alcanzar una mayor calidad de vida, en términos cualitativos y cuantitativos (Martínez de Carrasquero, 2020).

\section{ECUADOR}

En cambio, la Universidad Católica de Córdoba y la Pontificia Universidad Católica del Ecuador incorporan el 59\% los sub-ejes de la RSU en su declaración de Misión institucional, es decir, en su razón 
de ser actual y por ende debieran estar implementando acciones que reflejen dicha orientación en su diario quehacer institucional como una realidad claramente observable (Quezada \& Rodríguez, 2019).

La Facultad de Ciencias Médicas de la Universidad de Guayaquil tiene un alto nivel de pertinencia y sus aportes cobran relevancia, pues inducen a nuevas miradas y esfuerzos institucionales, básicamente en lo que tiene que ver con sus procesos de gestión, la tendencia a transformarse en facultad emprendedora e innovadora; pero de manera especial, debe buscar mecanismos y experiencias específicas que les ayuden a mejorar sus procesos de gestión en función del aporte a la Sociedad (Dáher et al., 2018).

\section{PERÚ}

Retomando la Ley Universitaria, el artículo 125 "Medios de promoción de la RSU” indica que las universidades implementarán la RSU, con reconocimiento de las instancias y miembros de la comunidad que lleven a cabo el proceso; y en una cuestión significativa para dicha política, obliga a un mínimo de inversión del presupuesto anual en esta materia para lo cual se establecerán mecanismos que incentiven su desarrollo por medio de proyectos de responsabilidad social, así como la creación de fondos concursables para estos efectos (Martí-Noguera, Calderón \& Fernández-Godenzi, 2018).

En USIL, por ejemplo, la sostenibilidad es uno de nuestros cuatro pilares transversales a todas las carreras, que fomentan actividades de responsabilidad social académicas, sociales y mucho más. Ello está inmerso también en la carrera de Administración, de la Facultad de Ciencias Empresariales, que permite que nuestros alumnos destaquen en el mundo empresarial como líderes, direccionando y organizando cualquier organización, incluyendo el propio emprendimiento, con claro foco en la sostenibilidad, además de la visión global e interés por la investigación (Javier, 2020).

\section{BRASIL}

Se ha verificado el alto grado de comprometimiento de esa Universidad Comunitaria con la causa social, con su desarrollo, con su inserción en prácticas, proyectos, acciones, actividades y en el enlace entre enseñanza, investigación y extensión, además de la preocupación de su dirección con la formación ciudadana (De Marco, Fanfa y Pinto, 2018).

Constituye una práctica que se desarrolla hace más de cinco años en la Facultad de Educación de la Universidad Federal de Minas Gerais (UFMG), en Belo Horizonte, Brasil. "GRUPEJA" es un grupo con registro formal institucionalizado, a través del Consejo Nacional de Desenvolvimiento Científico y Tecnológico (CNPq), que realiza tareas y actividades que responden a objetivos de trabajo con la sociedad, como parte de los vínculos universitarios y con la educación (González, Escalante \& Soares, 2021). 


\section{BOLIVIA}

En el marco de las actividades de Responsabilidad Social Universitaria, la Carrera de Comunicación junto a Sacha Forest y la Unión de Periodista Ambientales de Bolivia, inician una campaña con el objetivo de reducir y sustituir el uso de bolsas plásticas. En el marco de las actividades se tienen programado un conjunto de talleres internos y externos a universidad, así como la difusión de una campaña de sensibilización a través de distintos medios de comunicación. La campaña tiene como lema: "DESEMBÓLSATE. Por un mundo con seres humanos en equilibrio consigo mismos y con el ambiente" (UPAL, 2021).

En un acto realizado en la Casa Grande del Pueblo decenas de jóvenes participaron en el lanzamiento de "BOLIVIA C- REACTIVA", convocatoria dirigida a universitarios de los últimos cursos o egresados de la academia para diseñar la estrategias de promoción y comercialización de unidades productivas PyMEs, a través del uso de medios digitales...con la presencia del presidente del Estado Plurinacional de Bolivia, Exmo. Luis Arce Catacora y el Ministro de Desarrollo Productivo y Economía Plural, Néstor Huanca Chura, además de los representantes de los diferentes rubros de artesanos y productores de todo el país (Univalle, 2021).

\section{URUGUAY}

Los resultados obtenidos evidencian importantes problemas de conciliación entre trabajo y familia, producto de las extensas jornadas de trabajo que en ocasiones han desembocado en conflictos familiares, situación agravada por condiciones de trabajo inadecuadas, especialmente en cuanto a la prevención de riesgos en el establecimiento escolar y al bajo nivel de las remuneraciones percibidas por los profesores (Gaete Quezada \& Henríquez, 2016).

En el caso de la Ingeniería Agronómica, si bien existe una oferta de formación privada, no tiene egresados aún, por lo que la Facultad de Agronomía de la Universidad de la República sigue siendo la única institución que ha formado a estos profesionales en el país. En consecuencia, corresponde al desempeño de estos profesionales una importante cuota de responsabilidad sobre los efectos inmediatos y los impactos en el medio ambiente, la economía y la sociedad toda, que deriva de las recomendaciones que realizan a terceros y de las decisiones que toman en el ejercicio de la profesión (Marisquirena, Iñigo \& Passarini, 2018).

\section{PARAGUAY}

Los resultados destacan la importancia de la Responsabilidad Social Universitaria al enfrentar nuevos retos en el escenario social, político y económico del país, uniendo sus funciones de docencia, 
investigación, gestión y extensión universitaria, incidiendo de esta manera en la transformación social del Paraguay (Pérez \& Calvo, 2021).

En resumen, en pleno siglo XXI, las universidades del Paraguay se presentan ante el reto de convertirse en observatorios de vigilancia continua que permita el empoderamiento del conocimiento, nuevas ideas y conceptos, que conlleven a resolver los problemas de la sociedad, con objetivos socialmente responsables, en un contexto socioeducativo que provoca a las universidades del Paraguay a ser protagonistas de los nuevos desafíos,...(Duarte de Krummel, 2018).

\section{ARGENTINA}

Con la finalidad de aportar a los estudios que sobre el tema se realizan en diferentes universidades, el proyecto "Responsabilidad Social Universitaria.Enfoques y prácticas en dos universidades latinoamericanas" se implementó en paralelo en la Facultad de Ciencias Económicas de la Universidad Nacional de la Patagonia San Juan Bosco de la República Argentina (FCE) y la Facultad de Ciencias de la Administración del Politécnico Colombiano Jaime Isaza Cadavid... Los resultados señalan la necesidad de modificar las prácticas de RSU y trabajar en su efectiva puesta en práctica (Kent et al., 2018).

El programa Amartya Sen también fue una clara experiencia de responsabilidad social universitaria a nivel nacional, que entre 2009 y 2018 formó, con una mirada ética, a estudiantes avanzados de todas las facultades de ciencias económicas del país (Repetto, 2019).

\section{CHILE}

El rol socialmente responsable adoptado por las universidades chilenas en el desarrollo empresarial,... Se concluye que sólo trece de sesenta universidades chilenas estén operando este tipo de iniciativas; esto implica una desconexión en la forma de ejercer la RSU; a pesar de los requisitos actuales de vinculación con el medio ambiente, en miras a lograr un mejor resultado en sus propios procesos de acreditación institucional (Vega, Rojas y Salazar, 2018).

Los resultados muestran una mayor frecuencia del pluralismo, la democracia y la equidad, mientras que respecto del comportamiento socialmente responsable se destacan la integridad, la excelencia y la libertad. El estudio concluye que las universidades analizadas poseen una identidad corporativa explícita e inteligible, que no incluye mayormente valores y principios de la responsabilidad social universitaria (Gaete, 2020).

La Asociación de Universidades Jesuitas de América Latina ha generado una Red de Responsabilidad Social Universitaria que continúa dando pasos en sus proyectos e iniciativas para hacer realidad un modelo de universidad comprometida con la realidad social (Jesuitas, 2016). 
La Red URSULA es una asociación compuesta por Instituciones de Educación Superior (IES) y sin fines de lucro, que se desarrolló gracias al impulso de la dirección Social de la CAF Banco de Desarrollo de América Latina. URSULA es un espacio que permite la discusión de diferentes actores de la sociedad civil, gobiernos, organismos internacionales entre otros que promueve un cambio profundo en el rol social, ambiental de las universidades, con propuestas innovadoras para que estas gestionen y ejerzan su Responsabilidad Social (Gutiérrez, 2021).

URSULA defiende un pacto por una Universidad Saludable, Solidaria y Sostenible que permita enseñar desde el ejemplo, formar desde la coherencia entre el discurso y los hechos, conocer desde la solución concreta de los problemas sociales y ambientales cotidianos de nuestros territories (URSULA, 2020).

Se observa un esfuerzo permanente en el desarrollo de la responsabilidad social de las universidades latinoamericanas, mediante la formación de redes, asociaciones, eventos académicos y priorización de la RSU en sus objetivos institucionales.

\section{CONCLUSIONES}

La responsabilidad social universitaria (RSU) es importante por su impacto en la sociedad. Debe formar parte de las estrategias universitarias latinoamericanas y ser protagonistas de la mejora social. La gestión universitaria actual no debe descuidar la RSU. Han surgido diversas redes y asociaciones latinoamericanas que sirven de impulso de la RSU, por lo que se recomienda una mayor integración de las universidades públicas y privadas, para crear sinergias. En cada país latinoamericano analizado, destacan algunas universidades que lideran la RSU y que deberían ser imitadas, por los resultados positivos que están logrando. Se recomienda concientizar y capacitar al personal docente y administrativo en la RSU, para lograr un mayor compromiso social. 


\section{REFERENCIAS}

Aldeanueva Fernández, Ignacio, \& Arrabal Sánchez, Gabriel. (2018). La comunicación y medición de la Responsabilidad Social Universitaria: Redes sociales y propuesta de indicadores. Revista Digital de Investigación en Docencia Universitaria, 12(1), 121-136. https://dx.doi.org/10.19083/ridu.12.626

Anchieta-Barrios, L. y Pernía, J. (2018). Responsabilidad social universitaria: una visión prospectiva desde las funciones de docencia, investigación y extensión en la Universidad Centroccidental Lisandro Alvarado. Ágora de Heterodoxias. Vol. 4, $\mathrm{N}^{\mathrm{o}}$ 1: Enero - Junio. http://bdigital2.ula.ve:8080/xmlui/654321/1747

Baca Lobera, A y Reyes Núñez, Grisell, R. R. E. (2019). Universidad de Puerto Rico: responsabilidad social más allá del aula. Revista Educación Superior Y Sociedad (ESS), 29(29), 97-120. Recuperado a partir de https://www.iesalc.unesco.org/ess/index.php/ess3/article/view/93

Banegas, L. (2018). Responsabilidad Social y Educación en Honduras. Paradigma Revista de investigación educativa 23(36):111. DOI: 10.5377/paradigma. v23i36.6490

Barrero, A. y Rosero, A. (2018). Estado del Arte sobre Concepciones de la Diversidad en el Contexto Escolar Infantil. Revista Latinoamericana de Educación Inclusiva, 2018, 12(1), 39-55 https://doi.org/10.4067/S0718-73782018000100004

Barreto Cruz, Miguel Alejandro, Guacaneme Duque, Natalia, Navarrete Pita, Yulexy, \& Obando Gil, Huberto. (2021). Percepciones y prácticas de Responsabilidad Social Universitaria: Un llamado a la coherencia. Revista Estudios del Desarrollo Social: Cuba y América Latina, 9(1), e19. Epub 01 de abril de 2021. Recuperado de http://scielo.sld.cu/scielo.php?script=sci_arttext\&pid=S230801322021000100019\&lng=es\&tlng=es.

Dáher Nader, Jorge Elías, Panunzio, Amelia Patricia, \& Hernández Navarro, Marlene Isabel. (2018). La investigación científica: una función universitaria a considerar en el contexto ecuatoriano. EDUMECENTRO, 10(4), 166-179. Recuperado de http://scielo.sld.cu/scielo.php?script=sci_arttext\&pid=S2077-28742018000400011\&lng=es\&tlng=es.

De Marco, R., Fanfa, D. y Pinto, M. (2018). Responsabilidad social universitaria: La perspectiva de los colaboradores en uma universidad comunitaria brasileña. Tendencias Pedagógicas $\mathrm{N}^{\mathrm{o}} 31$. Recuperado de https://dialnet.unirioja.es/descarga/articulo/6383460.pdf

Duarte de Krummel, M. (2018). Las universidades del Paraguay y su influencia en la transformación social. Revista Científica de la UCSA, 5(3), 64-68. https://dx.doi.org/10.18004/ucsa/24098752/2018.005(03)064-068

Duque, Pedro, \& Cervantes-Cervantes, Luis-Salvador. (2019). Responsabilidad Social Universitaria: una revisión sistemática y análisis bibliométrico. Estudios Gerenciales, 35(153), 451-464. https://doi.org/10.18046/j.estger.2019.153.3389

Forero-Jiménez, M. Y. (2019). Modelo de responsabilidad social universitaria: una propuesta para las instituciones colombianas. Revista de Investigación, Desarrollo e Innovación, 9(2), 249-260. https://doi.org/10.19053/20278306.v9.n2.2019.9160 
Gaete Quezada, Ricardo. (2020). Dirección por valores y responsabilidad social en universidades estatales chilenas. Revista Digital de Investigación en Docencia Universitaria, 14(1), e1073. https://dx.doi.org/10.19083/ridu.2020.1073

Gaete Quezada, Ricardo, \& Henríquez, Fabián (2016). Análisis de la labor docente desde la perspectiva de la responsabilidad social interna. Cuadernos de Investigación Educativa, 7(2),13-29. ISSN: 15102432. Disponible en: https://www.redalyc.org/articulo.oa?id=443649571002

Gómez, Lina M, Alvarado Naveira, Yanitzary, \& Pujols Bernabel, Aileen. (2018). Implementing University Social Responsibility in the Caribbean: Perspectives of Internal Stakeholders. Revista Digital de Investigación en Docencia Universitaria, 12(1), 101-120. https://dx.doi.org/10.19083/ridu.12.714

González González, G. R., \& Fernández- Larrea, M. G. (2021). La responsabilidad social de la universidad: antecedentes, conceptos, tendencias y retos de la extensión universitaria. Revista Compromiso Social, 1(1), 11-22. Recuperado de https://revistacompromisosocial.unan.edu.ni/index.php/CompromisoSocial/article/view/18 (Original work published 30 de junio de 2019)

González Espino, Yaneisy. (2017). ¿Cómo evaluar la competencia investigativa desde la responsabilidad social universitaria?. Revista Cubana de Educación Superior, 36(2), 4-13. Recuperado de http://scielo.sld.cu/scielo.php?script=sci_arttext\&pid=S0257-43142017000200001\&lng=es\&tlng=es.

González Peña, M., Escalante Plá, L., \& Soares, L. (2021). Confluencias teóricas entre extensión, aprendizaje-servicio y responsabilidad social. Buenas prácticas universitarias. Masquedós - Revista De Extensión Universitaria, 6(6), 12. Recuperado a partir de http://ojs.extension.unicen.edu.ar/index.php/masquedos/article/view/123

Gutiérrez, E. (2021). Ética y responsabilidad social universitaria. Recuperado de https://www.uned.ac.cr/acontecer/a-diario/gestion-universitaria/4546-etica-y-responsabilidad-socialuniversitaria

Herrera Céspedes, F. (2019). Percepción De Las Dimensiones De Responsabilidad Social Universitaria (RSU) De Parte Del Grupo De Interés Interno De La Universidad De Costa Rica (UCR). Revista Nacional De Administración, 10(2), 61-75. https://doi.org/10.22458/rna.v10i2.2731

Ibarra Uribe, Luz Marina, Fonseca Bautista, César Darío, \& Santiago García, Rosana. (2020). La responsabilidad social universitaria. Misión e impactos sociales. Sinéctica, (54), e1008. Epub 07 de agosto de 2020.https://doi.org/10.31391/s2007-7033(2020)0054-011

Inmaculada Alonso Carrillo, Montserrat Núñez Chicharro y Abril E. Méndez (2019). Propuesta de indicadores de desempeño en materia de responsabilidad social universitaria aplicados a la Universidad de Panamá", Revista Contribuciones a las Ciencias Sociales, (mayo 2019). En línea: https://www.eumed.net/rev/cccss/2019/05/indicadores-desempeno-responsabilidad.html //hdl.handle.net/20.500.11763/cccss1905indicadores-desempeno-responsabilidad

Javier, J. (2020). La importancia de la responsabilidad social universitaria en las empresas. Recuperado de https://facultades.usil.edu.pe/ciencias-empresariales/la-importancia-de-la-responsabilidad-socialuniversitaria-en-las-empresas/ 
Jesuitas (2016). $6^{\circ}$ Encuentro de la Red de Responsabilidad Social Universitaria. Recuperado de http://jesuitasaru.org/6-encuentro-de-la-red-de-responsabilidad-social-universitaria/

Kent, Patricia; Trucco, Gabriel; Cifuentes Valenzuela, Araceli; Sendín, María Elena; Capart, Denisse; Capart, Susana; Domínguez, Rodrigo; Echeverría, Virginia; Pugh, Daniel; Salazar, Luis y Soetbeer, Germán (2018). Responsabilidad Social Universitaria: una mirada endógena. FACES, 24(51), 77-91. ISSN 0328-4050

Lorena, L. (2019). Responsabilidad social universitaria: ventaja competitiva de la de la Universidad de Costa Rica. Revista Educación Superior Y Sociedad (ESS), 29(29), 71-95. Recuperado de https://www.iesalc.unesco.org/ess/index.php/ess3/article/view/92

Llano Zhinin, Gladys Verónica, Carlozama Puruncajas, Jorge Fernando, Tipán Cañaveral, Daniel Fernando, \& Tipán Cañaveral, Gustavo Bernardo. (2021). La internacionalización en los procesos universitarios: un análisis estructural para universidades de Latinoamérica. Mendive. Revista de Educación, 19(1), 6-15. Epub 02 de marzo de 2021. Recuperado de http://scielo.sld.cu/scielo.php?script=sci_arttext\&pid=S1815-76962021000100006\&lng=es\&tlng=es.

Marisquirena Sebrango, Gustavo María, Iñigo Bajos, Enrique, \& Passarini, José. (2018). The Training and Job Performance of Agronomists in Uruguay. Revista Cubana de Educación Superior, 37(2), 131140. Recuperado en 18 de diciembre de 2021, de http://scielo.sld.cu/scielo.php?script=sci_arttext\&pid=S0257-43142018000200010\&lng=es\&tlng=en.

Marsiske, R. \& Vera de Flachs, C. (2018). A cien años de la Reforma Universitaria de Córdoba de 1918 y su proyección a Latinoamérica. Revista Historia de la Educación Latinoamericana, 20(30), 7-10. https://doi.org/10.19053/01227238.8025

Martí-Noguera, Juan-José, Calderón, Adolfo-Ignacio, \& Fernández-Godenzi, Adriana. (2018). La responsabilidad social universitaria en Iberoamérica: análisis de las legislaciones de Brasil, España y Perú. Revista iberoamericana de educación superior, 9(24), 107-124. https://doi.org/10.22201/iisue.20072872e.2018.24.264

Martínez de Carrasquero, C. (2020). Responsabilidad social universitaria, transferencia tecnológica y desarrollo endógeno. Estrategias de vinculación comunitaria. Revista Latinoamericana De Difusión Científica, $1(1), \quad 55-67 . \quad$ Recuperado de http://difusioncientifica.info/index.php/difusioncientifica/article/view/5

Medina Peña, Rolando, Franco Gómez, María, Torres Barreiro, Leodanis, Velázquez Rodríguez, Kerslin, Valencia Vera, Marina, \& Valencia Vera, Ana. (2017). La responsabilidad social universitaria en la actual sociedad del conocimiento. Un acercamiento necesario: a necessary approach. MediSur, 15(6), 786-791. Recuperado de http://scielo.sld.cu/scielo.php?script=sci_arttext\&pid=S1727897X2017000600006\&lng=es\&tlng=es.

Méndez Chang, A. (2020). Política de formación académica con enfoque de RSU para la Universidad de Panamá, planificación para la acción. Acción Y Reflexión Educativa, (45), 28-59. https://doi.org/10.48204/j.are.n45a2

Mosquera-Abadía, Henry Alberto, \& Carvajal-Ordoñez, Venus Flor Marina. (2021). Interacción universidad sociedad a través de la función de extensión. Entramado, 17(1), 186-203. Epub May 05, 2021.https://doi.org/10.18041/1900-3803/entramado.1.7230 
Nieto Gassos, K. D., \& Gazca Herrera, L. A. (2021). Acciones de Responsabilidad Social Universitaria en la oferta educativa del Área Económico-Administrativa de la Universidad Veracruzana. Revista GEON (Gestión, Organizaciones Y Negocios), 8(1), 1-12. https://doi.org/10.22579/23463910.209

Pérez Velilla, M. A. \& Calvo, G. (2021). Percepción de estudiantes de medicina acerca de la responsabilidad social universitaria. Revista Científica Estudios E Investigaciones, 10(1), 97-106. https://doi.org/10.26885/rcei.10.1.97

PUCMM (2019). PUCMM ingresa a Red de Responsabilidad Social Universitaria de ODUCAL. Recuperado de https://www.pucmm.edu.do/noticias/Lists/EntradasDeBlog/Post.aspx?ID=1640

Quezada, Ricardo Gaete, \& Rodríguez, Juliana Álvarez. (2019). Responsabilidad social universitaria en Latinoamérica. Los casos de URSULA y AUSJAL. Actualidades Investigativas en Educación, 19(3), 233-262. https://dx.doi.org/10.15517/aie.v19i3.38637

RED COMUNICA (2021). UASD participa en seminario sobre la "Responsabilidad Social Universitaria". Recuperado de https://redcomunica.csuca.org/index.php/universidad-autonoma-desanto-domingo-uasd/uasd-participa-en-seminario-sobre-la-responsabilidad-social-universitaria/

Repetto, J. (2019). La responsabilidad social llega a las universidades. Recuperado de http://saldoafavor.economicas.uba.ar/la-responsabilidad-social-llega-a-las-universidades/

Serna, Aura González, Gomes, Edvânia Torres Aguiar y Silva, Dweison Nunes Souza (2018). Puntuando algunos de los desafíos de las universidades latinoamericanas en el futuro presente1 1 Este artículo hace parte de investigaciones resultantes de la cooperación internacional entre la Universidade Federal de Pernambuco (UFPE), Brasil y Universidad Pontificia Bolivariana (UPB), Colombia, específicamente, por medio de los programas de pos grado en Desarrollo y Medio Ambiente - PRODEMA - UFPE y Maestría en Desarrollo - UPB, bien como de los Grupos de investigación Sociedade e Natureza Nexus UFPE y Territorio - UPB. . Avaliação: Revista da Avaliação da Educação Superior (Campinas) [online]. 2018, v. 23, n. 3, pp. 648-664. Disponible en: 〈https://doi.org/10.1590/S1414-40772018000300005>

UNICA (2020). Responsabilidad Social Universitaria. Recuperado de https://unica.edu.ni/responsabilidad-social-universitaria/

Univalle (2021). Univalle Participa En La Reactivación De Bolivia. Recuperado de http://www.univalle.edu/cochabamba/es/noticia/univalle-participa-en-la-reactivacion-de-bolivia

Universidad Pedagógica de El Salvador (2021). Mesa de Responsabilidad Social Universitaria. Recuperado de http://www.pedagogica.edu.sv/2021/07/29/mesarsu/

Universidad Rafael Landívar (2020). La Responsabilidad Social Universitaria la hacemos todos. Recuperado de https://principal.url.edu.gt/integracion-universitaria/rsu/la-responsabilidad-socialuniversitaria-la-hacemos-todos/

UPAL (2021). CAMPAÑA “DESEMBÓLSATE”. Recuperado de https://upal.edu.bo/desembolsate/ UPOLI (2016). Universitarios afirman que la política implica una gran responsabilidad social. Recuperado de https://www.upoli.edu.ni/noticias/ver/531-universitarios-afirman-que-la-politicaimplica-una-gran-responsabilidad-social

URSULA (2020). Quienes somos. Recuperado de https://unionursula.org/nosotros/quienes-somos/ 
Valdés Pérez, Miguel Gerardo, \& Villegas Rodríguez, Tania. (2017). Responsabilidad social universitaria: fundamento para la gestión sociocultural. Revista Cubana de Educación Superior, 36(3), 55-62. Recuperado de http://scielo.sld.cu/scielo.php?script=sci_arttext\&pid=S0257$43142017000300005 \& \operatorname{lng}=\mathrm{es} \& \ln \mathrm{ln}=\mathrm{es}$.

Vargas, Y. (2021). Proponen redefinir la función social de las universidades e institucionalizar la Responsabilidad Social Universitaria (RSU). Recuperado de https://presencia.unah.edu.hn/noticias/proponen-redefinir-la-funcion-social-de-las-universidades-consu-entorno-e-institucionalizar-la-responsabilidad-social-universitaria-rsu/

Vega, A., Rojas, María J. y Salazar, Guido (2018). Responsabilidad Social Universitaria: Aportes recientes al Desarrollo de las Empresas de Menor Tamaño en Chile. Revista Venezolana de Gerencia, vol. Esp, núm. 1, pp. 328-345. Recuperado de https://www.redalyc.org/journal/290/29062781019/html/ 